\title{
全氟烷基取代的芴基炔膦酸二乙酯化合物的一锅法合成
}

\author{
孙小莉 $^{a}$ 孙振华 ${ }^{a}$ 韩敏敏 ${ }^{b}$ 韩 靖 ${ }^{a}$ 何伟民 ${ }^{a}$ 陈 杰 ${ }^{a}$ \\ 邓红梅 ${ }^{c}$ 䂙 敏 ${ }^{c}$ 张 慧*,a,c 曹卫国*,a,d,e \\ $\left({ }^{a}\right.$ 上海大学化学系 上海 200444) \\ ( ${ }^{b}$ 上海大学钱伟长学院 上海 200444) \\ ( ${ }^{c}$ 上海大学微结构重点实验室 分析测试中心 上海 200444) \\ ( ${ }^{d}$ 中国科学院上海有机化学研究所有机金属化学国家重点实验室 上海 200032) \\ ( ${ }^{e}$ 中国科学院上海有机化学研究所 有机氟化学重点实验室 上海 200032)
}

\begin{abstract}
摘要 报道了一种以䒢酮、丙二腈、全氟烷基炔膦酸二乙酯为原料合成全氟烷基取代的芴基炔膦酸二乙酯类化合物的 新方法. 采用一锅合成法, 可将不同基团取代的蒒酮直接转化为一系列相应的全氟烷基取代的芴基炔膦酸二乙酯衍生 物. 整个反应过程无需金属催化, 具备反应条件温和、区域选择性好、产率较高、操作简便等优点.
\end{abstract}

关键词＼cjkstart多组分反应; 一锅法合成; 全氟烷基炔膦酸二乙酯; 芴类衍生物

\section{Synthesis of Perfluoroalkylated Fluorenes with Phosphonate Group via One-Pot Synthesis Process}

\author{
Sun, Xiaoli ${ }^{a} \quad$ Sun, Zhenhua ${ }^{a} \quad$ Han, Minmin $^{b} \quad$ Han, Jing ${ }^{a} \quad$ He, Weimin ${ }^{a} \quad$ Chen, Jie $^{a}$ \\ Deng, Hongmei ${ }^{c} \quad$ Shao, $\mathrm{Min}^{c} \quad$ Zhang, Hui*,a,c Cao, Weiguo ${ }^{*, a, d, e}$ \\ ( ${ }^{a}$ Department of Chemistry, Shanghai University, Shanghai 200444) \\ ( ${ }^{b}$ Qianweichang College, Shanghai University, Shanghai 200444) \\ ( ${ }^{c}$ Laboratory for Microstructures, Instrumental Analysis and Research Center of Shanghai University, Shanghai 200444) \\ ( ${ }^{d}$ State Key Laboratory of Organometallic Chemistry, Shanghai Institute of Organic Chemistry, \\ Chinese Academy of Sciences, Shanghai 200032) \\ ( ${ }^{e}$ Key Laboratory of Organofluorine Chemistry, Shanghai Institute of Organic Chemistry, Chinese \\ Academy of Sciences, Shanghai 200032)
}

\begin{abstract}
A simple and versatile metal-free protocol for the synthesis of perfluoroalkyated fluorene derivatives bearing phosphonate group is achieved. The reactions proceeded via one-pot three-component condensation of indenones, fluorinated alkynylphosphonates and malononitrile under mild conditions. This protocol is highly regioselective, operationally facile and applicable to a variety of indenones in good to excellent yields.

Keywords multicomponent reactions (MCRs); one-pot synthesis; perfluoroalkynyl phosphonate; fluorene
\end{abstract}

芴类衍生物在生物医药和光电材料领域所具备的 重要性使化学家对这类化合物产生了浓厚的兴趣 ${ }^{[1,2]}$. 近些年来芴在有机电致发光材料领域中得到了广泛的 研究与开发, 通过对芴类单元的修饰可以改变最高占有 分子轨道(HOMO)与最低未占分子轨道(LUMO)之间的
能隙差，从而改变发光波长，制作不同的发光器件 ${ }^{[3]}$ (图 1). 苯芴醇，作为一种合成的芴乙醇类化合物，具有较 强的灭无性体作用，用于治疗恶性症，具有疗效较高而 毒副反应较小的特点 ${ }^{[4]}$ (图 1). 此外, 荡类化合物在有机 金属化学中可以作为有效配体和金属络合形成新的配

\footnotetext{
* Corresponding authors. E-mail: wgcao@staff.shu.edu.cn; yehao7171@shu.edu.cn

Received August 24, 2018; revised September 13, 2018; published online October 19, 2018.

Dedicated to Professor Qingyun Chen on the occasion of his 90th birthday.

Project supported by the National Natural Science Foundation of China (No. 21672138).

国家自然科学基金(No. 21672138)资助项目.
} 
位体系, 在肽合成化学中则可以作为独特的保护基团而 得到广泛应用 ${ }^{[5]}$.
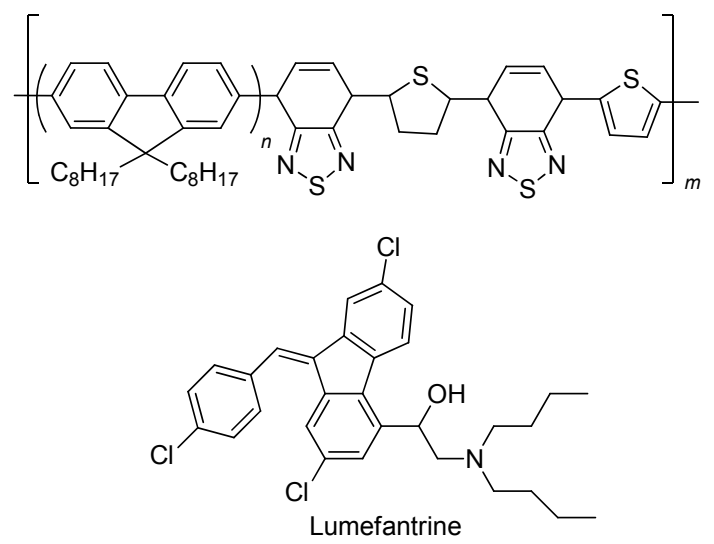

图 1 芴类的发光材料与苯芴醇

Figure 1 Light-emitting material of fluorene and lumefantrine

基于此类化合物的重要性，发展实用有效的合成种 类繁多的多官能团取代的芴类化合物的方法就成为有 机化学家需要迫切解决的一个重要问题. 传统构建芴环 的方法包括 Friedel-Crafts 型反应 ${ }^{[6]}$ 、过渡金属催化的环 合反应 ${ }^{[7]}$ 、自由基介入的环合反应 ${ }^{[8]}$ 和其他反应 ${ }^{[9]}$ 等.

传统方法虽然提供了合成芴类化合物的多种途径, 但是它们存在的一些缺点, 如原料难得、反应区域选择 性差、能进行反应的底物范围比较狭窄、反应条件苛刻、 多步骤合成等, 使得发展新的高效、底物范围广、反应 条件温和的合成方法成为有机化学家必须解决的难题.

多组分反应(MCRs)基于它们在有机合成中体现出 来的原子经济高效的特点, 现在已成为有机化学、药物 化学和组合化学领域中构建化合物环骨架的一个强有 力的工具 ${ }^{[10]}$.

我们课题组 ${ }^{[11]}$ 已经证实, 采用䒢酮、丙二腈和含氟 炔酸酯三组分一锅串联反应可以直接构建芴环, 得到全 氟烷基取代的芴衍生物. 第二步环合反应无需金属催 化, $80{ }^{\circ} \mathrm{C}$, 在碳酸钾存在下通过区域选择性 Michael 加 成环合等串联反应以较高产率得到目标产物.

研究证实, 当向生物活性分子中引入含氟基团或膦 酯基团后, 新形成的分子通常都会在脂溶性和酶的结合 性等物理化学性质方面产生显著变化 ${ }^{[12]}$. 由此我们设 想如果将含氟基团和膦酯基团一起引入生物活性分子, 所合成的这类新化合物对于发现新生物活性分子一定 具有重要意义. 于是我们尝试将孙振华等的方法做了拓 展, 用含氟炔膦酸二乙酯取代含氟炔酸酯, 一锅两步三 组分合成了全氟烷基取代的芴基炔膦酸二乙酯类化合 物, 反应同样无需金属催化, 环合反应在室温即可进行, 收率良好; 卤素原子、甲氧基和甲基等基团不受反应条 件影响，从而使底物范围得到一定拓展.

\section{1 结果与讨论}

首先，选定狮酮(1a)、丙二腈(2)与 4,4,4-三氟-2-炔 膦酸二乙酯 $(3 a)$ 的反应作为模板反应对反应条件进行优 化. 反应以一锅两步的方式进行. $1 \mathrm{a}$ 和 2 通过 Knoevenagel 缩合反应得到中间体 2-(2,3-二氢-1 $H$-狮-1亚基)丙二腈(5a), 后者与全氟烷基-2-炔膦酸二乙酯(3a) 及 $\mathrm{NaOH}$ 在 $25{ }^{\circ} \mathrm{C}$ 条件下继续反应 $6 \mathrm{~h}$, 得到所需的芴产 物 4a, 产率 61\%(表 1, Entry 1). 为了提高收率，我们对 影响第二步反应的几个要素包括碱、溶剂、碱量、反应 温度和反应时间等进行了进一步篎选(表 1). 对碱篎选 的结果表明 $\mathrm{NaOH}$ 最适合本反应，而且，第二步反应如 果没有碱存在, 得不到目标产物(表 1, Entry 8). 和甲苯 相比, 反应在其他溶剂如乙腈 $(\mathrm{MeCN})$ 、二氯甲烷

表 1 反应条件的优化 ${ }^{a}$

Table 1 Optimization of reaction conditions

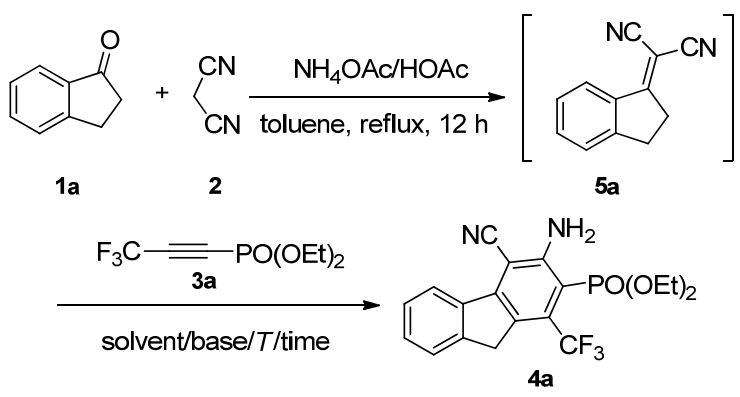

\begin{tabular}{|c|c|c|c|c|c|}
\hline Entry & Solvent & Base (equiv.) & $T^{b} /{ }^{\circ} \mathrm{C}$ & Time $^{c} / \mathrm{h}$ & Yield $^{d} / \%$ \\
\hline 1 & Toluene & $\mathrm{NaOH}(0.5)$ & r.t. & 6 & 61 \\
\hline 2 & Toluene & $\mathrm{KOH}(0.5)$ & r.t. & 6 & 57 \\
\hline 3 & Toluene & $\mathrm{K}_{2} \mathrm{CO}_{3}(0.5)$ & r.t. & 6 & 49 \\
\hline 4 & Toluene & Piperidine (0.5) & r.t. & 6 & - \\
\hline 5 & Toluene & $\mathrm{CsCO}_{3}(0.5)$ & r.t. & 6 & 45 \\
\hline 6 & Toluene & $\operatorname{CsF}(0.5)$ & r.t. & 6 & Trace \\
\hline 7 & Toluene & $\mathrm{Et}_{3} \mathrm{~N}(0.5)$ & r.t. & 6 & Trace \\
\hline 8 & Toluene & $\mathrm{NaOH}(0)$ & r.t. & 6 & - \\
\hline 9 & $\mathrm{MeCN}$ & $\mathrm{NaOH}(0.5)$ & r.t. & 6 & 52 \\
\hline 10 & DCM & $\mathrm{NaOH}(0.5)$ & r.t. & 6 & 55 \\
\hline 11 & THF & $\mathrm{NaOH}(0.5)$ & r.t. & 6 & 53 \\
\hline 12 & $\mathrm{EtOH}$ & $\mathrm{NaOH}(0.5)$ & r.t. & 6 & 53 \\
\hline 13 & Toluene & $\mathrm{NaOH}(1.0)$ & r.t. & 6 & 65 \\
\hline 14 & Toluene & $\mathrm{NaOH}(1.5)$ & r.t. & 6 & 69 \\
\hline 15 & Toluene & $\mathrm{NaOH}(2.0)$ & r.t. & 6 & 59 \\
\hline 16 & Toluene & $\mathrm{NaOH}(1.5)$ & 0 & 6 & 29 \\
\hline 17 & Toluene & $\mathrm{NaOH}(1.5)$ & 50 & 6 & 41 \\
\hline 18 & Toluene & $\mathrm{NaOH}(1.5)$ & r.t. & 12 & 73 \\
\hline 19 & Toluene & $\mathrm{NaOH}(1.5)$ & r.t. & 16 & 82 \\
\hline 20 & Toluene & $\mathrm{NaOH}(1.5)$ & r.t. & 24 & 81 \\
\hline
\end{tabular}

${ }^{a}$ Reaction conditions: 1a $(1.0 \mathrm{mmol}), \mathbf{2 a}(1.0$ equiv. $), \mathrm{AcOH}(0.1$ equiv.) and $\mathrm{NH}_{4} \mathrm{OAc}(0.2$ equiv.) were mixed and stirred in refluxing toluene $(5.0 \mathrm{~mL})$ for $12 \mathrm{~h}$. After cooling to the confined temperature, $3 \mathbf{a}$ (1.2 equiv.) and base were added and continued to be stirred for the referred time. ${ }^{b}$ Reaction temperature for the second step. ${ }^{c}$ Reaction time for the second step. ${ }^{d}$ Isolated yields for two steps in one pot. 
$(\mathrm{DCM}) 、$ 四氢呋喃(THF)和无水乙醇 $(\mathrm{EtOH})$ 中进行时, 所 得产物收率略低(表 1 , Entry 1 vs. Entries 9 12). 将 $\mathrm{NaOH}$ 的量从 0.5 equiv. 增加到 1.5 equiv., 产率提高到 69\%(表 1, Entry 14), 但是进一步增加碱的用量, 收率反 而降低了(表 1 , Entry 15). 将反应置于 $0{ }^{\circ} \mathrm{C}$ 或者 $50{ }^{\circ} \mathrm{C}$ 进 行, 导致反应收率下降明显(表 1 , Entries 16 和 17). 将第 二步反应时间由 $6 \mathrm{~h}$ 延长到 $16 \mathrm{~h}$ 后, 得到 $82 \%$ 收率的 $\mathbf{4 a}$ (表 1, Entry 19), 进一步延长反应时间，收率则再没有 显著提升(表 1, Entry 20).

利用得到的最优化反应条件(表 1 , Entry 19), 我们 对狮酮上取代基 $\mathrm{R}$ 的性质和位置对反应的影响做了探 索. 如表 2 所示, $\mathrm{R}$ 为给电子基团或吸电子基团取代的狮 酮都适用于该反应，均能以好的收率得到所需的全氟烷 基芴基炔膦酸二乙酯衍生物. 在同一个取代位置上, 取 代基的吸电子能力越强, 收率降低越明显(表 2 , Entry 5 vs. Entries 4 和 6), 总的来说, 未取代狮酮和给电子取代

表 2 全氟烷基芴基炔膦酸二乙酯的合成 ${ }^{a}$

Table 2 Scope of the synthesis of perfluoroalkylated fluorenes with phosphonate group
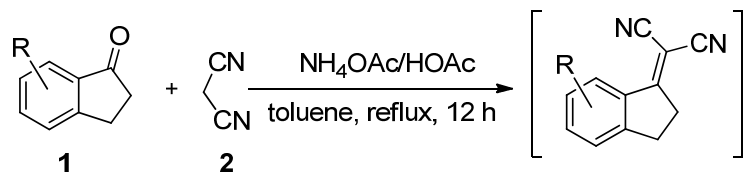

$5 a$

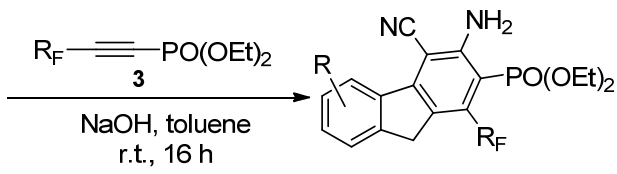

$4 a$

\begin{tabular}{cllcc}
\hline Entry & \multicolumn{1}{c}{$\mathrm{R}$} & \multicolumn{1}{c}{$\mathrm{R}_{\mathrm{F}}$} & Product $\mathbf{4}$ & Yield $^{b} \%$ \\
\hline 1 & $\mathrm{H}$ & $\mathrm{CF}_{3}$ & $\mathbf{4 a}$ & 82 \\
2 & $6-\mathrm{Br}$ & $\mathrm{CF}_{3}$ & $\mathbf{4 b}$ & 57 \\
3 & $4-\mathrm{Br}$ & $\mathrm{CF}_{3}$ & $\mathbf{4 c}$ & 50 \\
4 & $5-\mathrm{Cl}$ & $\mathrm{CF}_{3}$ & $\mathbf{4 d}$ & 62 \\
5 & $5-\mathrm{F}$ & $\mathrm{CF}_{3}$ & $\mathbf{4 e}$ & 48 \\
6 & $5-\mathrm{Br}$ & $\mathrm{CF}_{3}$ & $\mathbf{4 f}$ & 68 \\
7 & $5-\mathrm{MeO}$ & $\mathrm{CF}_{3}$ & $\mathbf{4 g}$ & 70 \\
8 & $4-\mathrm{Me}$ & $\mathrm{CF}_{3}$ & $\mathbf{4 h}$ & 74 \\
9 & $5-\mathrm{Me}$ & $\mathrm{CF}_{3}$ & $\mathbf{4 i}$ & 70 \\
10 & $6-\mathrm{Me}$ & $\mathrm{CF}_{3}$ & $\mathbf{4 j}$ & 75 \\
11 & $6-\mathrm{MeO}$ & $\mathrm{CF}_{3}$ & $\mathbf{4 k}$ & 78 \\
12 & $\mathrm{H}$ & $\mathrm{C}_{2} \mathrm{~F}_{5}$ & $\mathbf{4 l}$ & 74 \\
13 & $5-\mathrm{Me}$ & $\mathrm{C}_{2} \mathrm{~F}_{5}$ & $\mathbf{4 m}$ & 60 \\
14 & $6-\mathrm{MeO}$ & $\mathrm{C}_{2} \mathrm{~F}_{5}$ & $\mathbf{4 n}$ & 67 \\
15 & $\mathrm{H}$ & $n-\mathrm{C}_{3} \mathrm{~F}_{7}$ & $\mathbf{4 o}$ & 58 \\
16 & $5-\mathrm{Me}$ & $n-\mathrm{C}_{3} \mathrm{~F}_{7}$ & $\mathbf{4 p}$ & 51 \\
17 & $6-\mathrm{MeO}$ & $n-\mathrm{C}_{3} \mathrm{~F}_{7}$ & $\mathbf{4 q}$ & 49 \\
\hline
\end{tabular}

${ }^{a}$ Reaction conditions: 1 (1.0 mmol), 2 (1.0 equiv.), $\mathrm{AcOH}(0.1$ equiv.) and $\mathrm{NH}_{4} \mathrm{OAc}(0.2$ equiv.) were stirred in refluxing toluene $(5.0 \mathrm{~mL})$ for $12 \mathrm{~h}$ After cooling to the room temperature, 3 (1.2 equiv.) and $\mathrm{NaOH}$ (1.5 equiv.) were added and continued to be stirred for $16 \mathrm{~h}$ at the same temperature. ${ }^{b}$ Isolated yields for two steps in one pot.
基能得到更高收率(表 2, Entry 1 和 Entries 7 11). 用氟、 氯和溴等卤素原子取代的苆酮, 均以良好的产率得到目 标产物，从而可以利用取代反应或者金属催化偶联反应 实现目标产物进一步的衍生官能化. 取代基在不同的取 代位置例如甲基在芴酮的 $4 、 5$ 或 6 位取代时，也可以以 良好的收率得到所需的苆基炔膦酸二乙酯衍生物(表 2 , Entries 8 10), 说明取代基取代位置对反应影响不大. 用碳链更长的五氟乙基或七氟正丙基替代三氟甲基取 代的炔膦酸二乙酯, 反应一样可以顺利进行, 并得到满 意收率，虽然因为位阻缘故，收率相比 $2 \mathrm{a}$ 有所降低(表 2, Entry 1 vs. Entries 12 和 15).

$4 \mathbf{a}$ 的结构由单晶 $\mathrm{X}$ 射线分析结果确定(图 2) ${ }^{[13]}$. 其 他产物的结构通过与 $\mathbf{4 a}$ 的 ${ }^{1} \mathrm{H}$ NMR, ${ }^{13} \mathrm{C}$ NMR, ${ }^{19} \mathrm{~F}$ NMR, IR 和 MS, HRMS 等进行比较后得到确认.

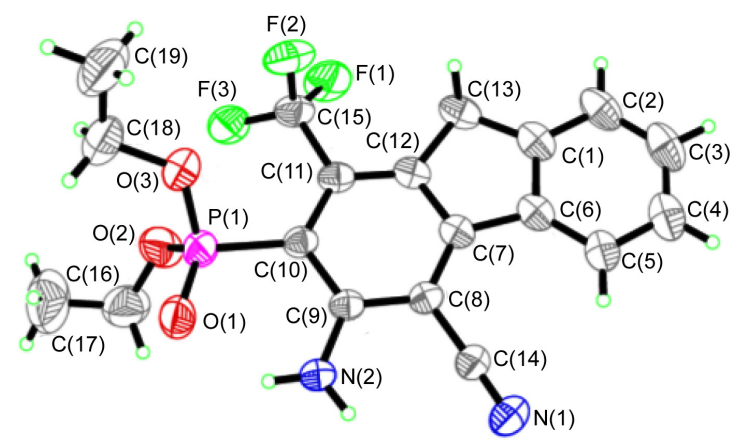

图 $24 \mathrm{a}$ 的 $\mathrm{X}$ 射线晶体结构

Figure 2 X-ray crystal structure of $4 a$

根据实验结果和以前相关的文献报道 ${ }^{[11,14]}$, 该反应 的反应历程如 Scheme 1 所示. 首先茚酮 1 和丙二腈(2) 通过 Knoevenagel 缩合反应得到中间体 2-(2,3-二氢- $1 H$ 狮-1-亚基)丙二腈(5) ${ }^{[15]}$, 然后中间体 $\mathbf{5}$ 先是在碱 $\mathrm{NaOH}$ 的作用下发生去质子化，得到碳负离子中间体 $\mathbf{6}$, 中间 体 6 高区域选择性地进攻全氟烷基-2-炔膦酸二乙酯 3 中 构建炔键的两个碳中亲核性更强的 2 位碳原子, 通过分 子间 Michael 加成反应形成新的碳负离子 7, 后者通过 对氰基进行分子内亲核加成以实现成环得到中间体 $\mathbf{8 , 8}$ 随之发生芳构化得到目标产物 4.

\section{2 结论}

发展了一种简单高效的合成全氟烷基苆基炔膦酸 二乙酯类化合物的新方法. 该方法通过一锅两步方式, 经过 Knoevenagel 缩合反应/Michael 加成反应/环化的一 系列过程将含氟基团和炔膦酸二乙酯基团一并引入到 芴分子中. 该反应的起始原料容易获得，实验操作简便， 不需要专门分离出第一步中间体，反应时间短，底物范 围广，具有被用来设计新药物和有机光电材料的良好应 


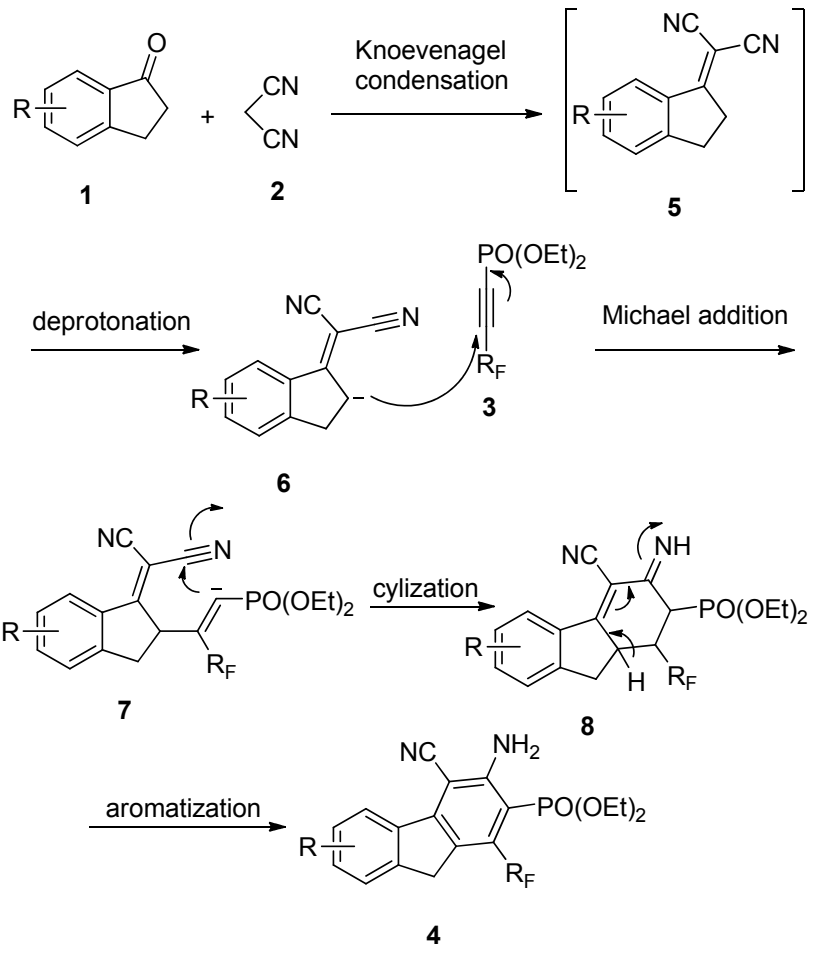

图式 1 可能的反应路径

Scheme 1 Possible reaction pathway

用前景.

\section{3 实验部分}

\section{1 仪器与试剂}

熔点在上海物理光学仪器公司生产的 WRS-1 数字 熔点仪上测得, 未经校正. ${ }^{1} \mathrm{H},{ }^{19} \mathrm{~F} N M R$ 和 ${ }^{13} \mathrm{C}$ NMR 光谱 在 Bruker AVANCE III HD-600 MHz, Bruker DRX-500 $\mathrm{MHz}$ 和 JNM-ECZ400S/L-400 MHz 光谱仪上测得, ${ }^{1} \mathrm{H}$ $\mathrm{NMR}$ 和 ${ }^{13} \mathrm{C} N \mathrm{NMR}$ 光谱以 $\mathrm{TMS}$ 为内标, ${ }^{19} \mathrm{~F} \mathrm{NMR}$ 以 $\mathrm{C}_{6} \mathrm{~F}_{6}$ 为内标, $\mathrm{CDCl}_{3}$ 为溶剂. IR 光谱在 AVATAR370 FTIR 光谱 仪上测得. 低分辨率质谱 (LR-MS) 和高分辨率质谱 (HR-MS) 分别在 Agilent 6230, Thermo Fisher Scientic LTQ FTICR-MS 或 Agilent 1100 LC/MSD SL 仪器上测 得. $\mathrm{X}$ 射线单晶结构测定分析在 Bruker Smart Apex2 $\mathrm{CCD}$ 光谱仪上进行. 文中报道的产率是指经柱层析分 离得到的化合物的产率, 其纯度通过 ${ }^{1} \mathrm{H}$ NMR 测定. 实 验中所用试剂未经标明的, 均为直接购买的分析纯试 剂, 使用前未做进一步纯化. 全氟烷基-2-炔膦酸二乙酯 根据参考文献[16]制备.

\section{2 实验方法}

茆酮 1 (1.0 mmol)、丙二腈 2 (1.0 equiv.)、冰醋酸 $(0.1$ equiv.) 和醋酸铵 $(0.2$ equiv. $)$ 的混合物在甲苯 $(5.0 \mathrm{~mL})$ 中 回流 $12 \mathrm{~h}$ 后, 将反应体系冷却至室温, 加入全氟烷基-2炔膦酸二乙酯 3 (1.2 equiv.) 和氢氧化钠(1.5 equiv.), 在室
温下继续摚拌 $16 \mathrm{~h}$. 循环水葲真空下除去溶剂, 残留物 经硅胶柱层析纯化, 用石油醚/乙酸乙酯 $(V: V, 15: 1$ $10: 1)$ 洗脱, 得到所需产物 4 .

3-氨基-4-氧基-1-(三氟甲基)- $9 H$-芴-2-炔膦酸二乙 酯(4a): 黄色固体. m.p. 142.2 142.7 ${ }^{\circ} \mathrm{C} ;{ }^{1} \mathrm{H}$ NMR (500 $\left.\mathrm{MHz}, \mathrm{CDCl}_{3}\right) \delta: 1.36(\mathrm{t}, J=7.3 \mathrm{~Hz}, 6 \mathrm{H}), 4.07(\mathrm{~s}, 2 \mathrm{H})$, $4.11 \sim 4.27$ (m, 4H), 7.15 (brs, $2 \mathrm{H}), 7.47 \sim 7.52(\mathrm{~m}, 2 \mathrm{H})$, $7.58 \sim 7.59(\mathrm{~m}, 1 \mathrm{H}), 8.49 \sim 8.50(\mathrm{~m}, 1 \mathrm{H}) ;{ }^{13} \mathrm{C}$ NMR $(125$ $\left.\mathrm{MHz}, \mathrm{CDCl}_{3}\right) \delta: 16.2\left(\mathrm{~d},{ }^{3} J_{\mathrm{C}-\mathrm{P}}=7.0 \mathrm{~Hz}\right), 37.5,63.0(\mathrm{~d}$, $\left.{ }^{2} J_{\mathrm{C}-\mathrm{P}}=6.0 \mathrm{~Hz}\right), 93.8\left(\mathrm{~d},{ }^{3} J_{\mathrm{C}-\mathrm{P}}=16.3 \mathrm{~Hz}\right), 103.6\left(\mathrm{~d},{ }^{1} J_{\mathrm{C}-\mathrm{P}}=\right.$ $183.0 \mathrm{~Hz}), 116.0,122.9,123.6\left(\mathrm{qd},{ }^{3} J_{\mathrm{C}-\mathrm{P}}=5.3 \mathrm{~Hz},{ }^{1} J_{\mathrm{C}-\mathrm{F}}=\right.$ $\left.275.1 \mathrm{~Hz}, \mathrm{CF}_{3}\right), 124.7,127.6,130.3 \sim 130.4(\mathrm{~m}), 130.5$, $134.0\left(\mathrm{qd},{ }^{2} J_{\mathrm{C}-\mathrm{P}}=5.1 \mathrm{~Hz},{ }^{2} J_{\mathrm{C}-\mathrm{F}}=32.4 \mathrm{~Hz}\right), 136.7,145.5$, 149.2, $155.7\left(\mathrm{~d},{ }^{2} J_{\mathrm{C}-\mathrm{P}}=10.0 \mathrm{~Hz}\right) ;{ }^{19} \mathrm{~F}$ NMR $(470 \mathrm{MHz}$, $\left.\mathrm{CDCl}_{3}\right) \delta:-54.9\left(\mathrm{~s}, \mathrm{CF}_{3}\right)$; IR (KBr) v: 3289, 2923, 2853, 2209, 1622, 1397, 1284, 1177, 1127, 1047, 1022, 766 $\mathrm{cm}^{-1}$; MS (ESI) $m / z(\%): 411[(\mathrm{M}+\mathrm{H})]^{+}$; HRMS (DART) calcd for $\mathrm{C}_{19} \mathrm{H}_{19} \mathrm{~F}_{3} \mathrm{~N}_{2} \mathrm{O}_{3} \mathrm{P}(\mathrm{M}+\mathrm{H})^{+}$411.1080, found 411.1079 .

氨基-6-澳-4-氮基-1-(三氟甲基)- $9 H$-芴-2-炔膦酸二 乙酯(4b): 黄色固体. m.p. $143.5 \sim 143.8{ }^{\circ} \mathrm{C} ;{ }^{1} \mathrm{H}$ NMR $\left(500 \mathrm{MHz}, \mathrm{CDCl}_{3}\right) \delta: 1.36(\mathrm{t}, J=7.0 \mathrm{~Hz}, 6 \mathrm{H}), 4.00(\mathrm{~s}, 2 \mathrm{H})$, $4.11 \sim 4.30(\mathrm{~m}, 4 \mathrm{H}), 7.17$ (brs, $2 \mathrm{H}), 7.42 \sim 7.44(\mathrm{~m}, 1 \mathrm{H})$, $7.58 \sim 7.60(\mathrm{~m}, 1 \mathrm{H}), 8.54 \sim 8.55(\mathrm{~m}, 1 \mathrm{H}) ;{ }^{13} \mathrm{C}$ NMR $(125$ $\left.\mathrm{MHz}, \mathrm{CDCl}_{3}\right) \delta: 16.2\left(\mathrm{~d},{ }^{3} J_{\mathrm{C}-\mathrm{P}}=7.0 \mathrm{~Hz}\right), 37.3,63.2(\mathrm{~d}$, $\left.{ }^{2} J_{\mathrm{C}-\mathrm{P}}=5.6 \mathrm{~Hz}\right), 93.9\left(\mathrm{~d},{ }^{3} J_{\mathrm{C}-\mathrm{P}}=16.3 \mathrm{~Hz}\right), 104.8\left(\mathrm{~d},{ }^{1} J_{\mathrm{C}-\mathrm{P}}=\right.$ $182.4 \mathrm{~Hz}), 115.7,121.7,123.5\left(\mathrm{qd},{ }^{3} J_{\mathrm{C}-\mathrm{P}}=5.2 \mathrm{~Hz},{ }^{1} J_{\mathrm{C}-\mathrm{F}}=\right.$ $\left.275.6 \mathrm{~Hz}, \mathrm{CF}_{3}\right), 125.9,126.2,130.6$ (m), 133.5, 134.4 (qd, $\left.{ }^{2} J_{\mathrm{C}-\mathrm{P}}=5.0 \mathrm{~Hz},{ }^{2} J_{\mathrm{C}-\mathrm{F}}=32.5 \mathrm{~Hz}\right), 138.8,144.2,147.7,155.8$ $\left(\mathrm{d},{ }^{2} J_{\mathrm{C}-\mathrm{P}}=10.5 \mathrm{~Hz}\right) ;{ }^{19} \mathrm{~F}$ NMR $\left(470 \mathrm{MHz}, \mathrm{CDCl}_{3}\right) \delta$ : $-55.0\left(\mathrm{~s}, \mathrm{CF}_{3}\right.$ ); IR (KBr) $\delta: 3264,2919,2851,2209,1619$, 1394, 1284, 1164, 1129, 1047, 1011, $776 \mathrm{~cm}^{-1}$; MS (ESI) $m / z: 489(\mathrm{M}+\mathrm{H})^{+}$; HRMS (ESI) calcd for $\mathrm{C}_{19} \mathrm{H}_{18^{-}}$ $\mathrm{BrF}_{3} \mathrm{~N}_{2} \mathrm{O}_{3} \mathrm{P}(\mathrm{M}+\mathrm{H})^{+}$489.0192, found 489.0187.

3 -氨基-8-溴-4-氧基-1-(三氟甲基)- $9 \mathrm{H}$-芴-2-炔膦酸 二乙酯(4c)：黄色固体. m.p. $182.5 \sim 182.8{ }^{\circ} \mathrm{C} ;{ }^{1} \mathrm{H}$ NMR $\left(500 \mathrm{MHz}, \mathrm{CDCl}_{3}\right) \delta: 1.36(\mathrm{t}, J=7.0 \mathrm{~Hz}, 6 \mathrm{H}), 4.00(\mathrm{~s}, 2 \mathrm{H})$, $4.11 \sim 4.30(\mathrm{~m}, 4 \mathrm{H}), 7.18$ (brs, $2 \mathrm{H}), 7.35 \sim 7.38(\mathrm{~m}, 1 \mathrm{H})$, $7.63 \sim 7.64(\mathrm{~m}, 1 \mathrm{H}), 8.40 \sim 8.42(\mathrm{~m}, 1 \mathrm{H}) ;{ }^{13} \mathrm{C}$ NMR $(125$ $\left.\mathrm{MHz}, \mathrm{CDCl}_{3}\right) \delta: 16.2\left(\mathrm{~d},{ }^{3} J_{\mathrm{C}-\mathrm{P}}=7.0 \mathrm{~Hz}\right), 39.3,63.2(\mathrm{~d}$, $\left.{ }^{2} J_{\mathrm{C}-\mathrm{P}}=5.6 \mathrm{~Hz}\right), 94.2\left(\mathrm{~d},{ }^{3} J_{\mathrm{C}-\mathrm{P}}=16.0 \mathrm{~Hz}\right), 104.9\left(\mathrm{~d},{ }^{1} J_{\mathrm{C}-\mathrm{P}}=\right.$ $182.2 \mathrm{~Hz}), 115.8,119.8,121.9,123.5\left(\mathrm{qd},{ }^{3} J_{\mathrm{C}-\mathrm{P}}=5.2 \mathrm{~Hz}\right.$, $\left.{ }^{1} J_{\mathrm{C}-\mathrm{F}}=275.6 \mathrm{~Hz}, \mathrm{CF}_{3}\right), 129.5,129.6(\mathrm{~m}), 133.4,134.5(\mathrm{qd}$, $\left.{ }^{2} J_{\mathrm{C}-\mathrm{P}}=5.0 \mathrm{~Hz},{ }^{2} J_{\mathrm{C}-\mathrm{F}}=32.4 \mathrm{~Hz}\right), 138.2,145.5,148.6,155.8$ $\left(\mathrm{d},{ }^{2} J_{\mathrm{C}-\mathrm{P}}=10.3 \mathrm{~Hz}\right) ;{ }^{19} \mathrm{~F}$ NMR $\left(470 \mathrm{MHz}, \mathrm{CDCl}_{3}\right) \delta$ : 
-54.9 (s, $\mathrm{CF}_{3}$ ); IR (KBr) v: 3276, 2909, 2854, 2210, 1622, 1394, 1284, 1170, 1135, 1038, 1016, $763 \mathrm{~cm}^{-1}$; MS (ESI) $m / z: 489[(\mathrm{M}+\mathrm{H})]^{+}$; HRMS (ESI) calcd for $\mathrm{C}_{19} \mathrm{H}_{18^{-}}$ $\mathrm{BrF}_{3} \mathrm{~N}_{2} \mathrm{O}_{3} \mathrm{P}(\mathrm{M}+\mathrm{H})^{+}$489.0192, found 489.0188.

3-氨基-4-氧基-8-甲氧基-1-(三氟甲基)- $9 H$-芴-2-炔 膦酸二乙酯 $(4 d)$ : 黄色固体. m.p. $145.6 \sim 146.1{ }^{\circ} \mathrm{C} ;{ }^{1} \mathrm{H}$ NMR (400 MHz, $\left.\mathrm{CDCl}_{3}\right) \delta: 1.34(\mathrm{t}, J=7.2 \mathrm{~Hz}, 6 \mathrm{H}), 3.88$ $(\mathrm{s}, 3 \mathrm{H}), 3.98(\mathrm{~s}, 2 \mathrm{H}), 4.07 \sim 4.26(\mathrm{~m}, 4 \mathrm{H}), 6.97 \sim 7.00(\mathrm{~m}$, $1 \mathrm{H}), 7.05 \sim 7.06(\mathrm{~m}, 1 \mathrm{H}), 7.08$ (brs, $2 \mathrm{H}), 8.33 \sim 8.35(\mathrm{~m}$, $1 \mathrm{H}) ;{ }^{13} \mathrm{C}$ NMR $\left(125 \mathrm{MHz}, \mathrm{CDCl}_{3}\right) \delta: 16.2\left(\mathrm{~d},{ }^{3} J_{\mathrm{C}-\mathrm{P}}=6.6\right.$ $\mathrm{Hz}), 37.4,55.7,62.9\left(\mathrm{~d},{ }^{2} J_{\mathrm{C}-\mathrm{P}}=5.6 \mathrm{~Hz}\right), 92.7$ (d, ${ }^{3} J_{\mathrm{C}-\mathrm{P}}=$ $16.4 \mathrm{~Hz}), 101.7\left(\mathrm{~d},{ }^{1} J_{\mathrm{C}-\mathrm{P}}=183.9 \mathrm{~Hz}\right), 109.6,114.3,116.3$, $123.7\left(\mathrm{qd},{ }^{3} J_{\mathrm{C}-\mathrm{P}}=5.0 \mathrm{~Hz},{ }^{1} J_{\mathrm{C}-\mathrm{F}}=275.5 \mathrm{~Hz}, \mathrm{CF}_{3}\right), 124.3$, $129.7,130.1 \sim 130.2(\mathrm{~m}), 133.6\left(\mathrm{qd},{ }^{2} J_{\mathrm{C}-\mathrm{P}}=5.2 \mathrm{~Hz},{ }^{2} J_{\mathrm{C}-\mathrm{F}}=\right.$ $32.5 \mathrm{~Hz}), 148.1,149.5,155.8\left(\mathrm{~d},{ }^{2} J_{\mathrm{C}-\mathrm{P}}=10.1 \mathrm{~Hz}\right), 162.1$; ${ }^{19} \mathrm{~F}$ NMR $\left(470 \mathrm{MHz}, \mathrm{CDCl}_{3}\right) \delta$ : $-54.9\left(\mathrm{~s}, \mathrm{CF}_{3}\right)$; IR $(\mathrm{KBr})$ $v$ : 3297, 3218, 2909, 2963, 2835, 2210, 1606, 1402, 1284, 1167, 1118, 1042, 1017, $782 \mathrm{~cm}^{-1}$; MS (ESI) m/z: $441[(\mathrm{M}+\mathrm{H})]^{+}$; HRMS (ESI) calcd for $\mathrm{C}_{20} \mathrm{H}_{21} \mathrm{~F}_{3} \mathrm{~N}_{2} \mathrm{O}_{4} \mathrm{P}$ $(\mathrm{M}+\mathrm{H})^{+}$441.1195, found 441.1189.

3-氨基-7-氯-4-氰基-1-(三氟甲基)- $9 H$-芴-2-炔膦酸 二乙酯(4e): 黄色固体. m.p. 155.9 156.4 ${ }^{\circ} \mathrm{C} ;{ }^{1} \mathrm{H} \mathrm{NMR}$ $\left(500 \mathrm{MHz}, \mathrm{CDCl}_{3}\right) \delta: 1.36(\mathrm{t}, J=7.0 \mathrm{~Hz}, 6 \mathrm{H}), 4.01(\mathrm{~s}, 2 \mathrm{H})$, $4.11 \sim 4.30(\mathrm{~m}, 4 \mathrm{H}), 7.16(\mathrm{brs}, 2 \mathrm{H}), 7.41 \sim 7.43(\mathrm{~m}, 1 \mathrm{H})$, $7.52 \sim 7.53(\mathrm{~m}, 1 \mathrm{H}), 8.34 \sim 8.35(\mathrm{~m}, 1 \mathrm{H}) ;{ }^{13} \mathrm{C}$ NMR $(125$ $\left.\mathrm{MHz}, \mathrm{CDCl}_{3}\right) \delta: 16.2\left(\mathrm{~d},{ }^{3} J_{\mathrm{C}-\mathrm{P}}=6.8 \mathrm{~Hz}\right), 37.3,63.1$ (d, $\left.{ }^{2} J_{\mathrm{C}-\mathrm{P}}=5.9 \mathrm{~Hz}\right), 93.7\left(\mathrm{~d},{ }^{3} J_{\mathrm{C}-\mathrm{P}}=16.2 \mathrm{~Hz}\right), 104.2\left(\mathrm{~d},{ }^{1} J_{\mathrm{C}-\mathrm{P}}=\right.$ $181.8 \mathrm{~Hz}), 115.9,123.6\left(\mathrm{qd},{ }^{3} J_{\mathrm{C}-\mathrm{P}}=5.4 \mathrm{~Hz},{ }^{1} J_{\mathrm{C}-\mathrm{F}}=275.3\right.$ $\left.\mathrm{Hz}, \mathrm{CF}_{3}\right), 123.9,125.1,128.2,130.0 \sim 130.1(\mathrm{~m}), 134.3$ $\left(\mathrm{qd}, \quad{ }^{2} J_{\mathrm{C}-\mathrm{P}}=5.0 \mathrm{~Hz},{ }^{2} J_{\mathrm{C}-\mathrm{F}}=32.8 \mathrm{~Hz}\right), 135.3,136.8,147.2$, 148.1, $155.8\left(\mathrm{~d},{ }^{2} J_{\mathrm{C}-\mathrm{P}}=10.1 \mathrm{~Hz}\right) ;{ }^{19} \mathrm{~F}$ NMR $(470 \mathrm{MHz}$, $\left.\mathrm{CDCl}_{3}\right) \delta:-55.0\left(\mathrm{~s}, \mathrm{CF}_{3}\right)$; IR (KBr) v: 3257, 2911, 2209, 1616, 1400, 1287, 1170, 1043, 1017, $768 \mathrm{~cm}^{-1}$; MS (ESI) $m / z: 445(\mathrm{M}+\mathrm{H})^{+}$; HRMS (ESI) calcd for $\mathrm{C}_{19} \mathrm{H}_{18} \mathrm{ClF}_{3}{ }^{-}$ $\mathrm{N}_{2} \mathrm{O}_{3} \mathrm{P}(\mathrm{M}+\mathrm{H})^{+}$445.0695, found 445.0690.

3-氨基-4-氭基-7-氟-1-(三氟甲基)- $9 H$-芴-2-炔膦酸 二乙酯(4f): 黄色固体. m.p. 179.8 180.2 ${ }^{\circ} \mathrm{C} ;{ }^{1} \mathrm{H} \mathrm{NMR}$ $\left(600 \mathrm{MHz}, \mathrm{CDCl}_{3}\right) \delta: 1.35(\mathrm{t}, J=6.9 \mathrm{~Hz}, 6 \mathrm{H}), 4.04(\mathrm{~s}, 2 \mathrm{H})$, $4.11 \sim 4.26$ (m, 4H), 7.16 (brs, 2H), $7.17 \sim 7.19(\mathrm{~m}, 1 \mathrm{H})$, $7.25(\mathrm{~s}, 1 \mathrm{H}), 8.42 \sim 8.44(\mathrm{~m}, 1 \mathrm{H}) ;{ }^{13} \mathrm{C}$ NMR $(125 \mathrm{MHz}$, $\left.\mathrm{CDCl}_{3}\right) \delta: 16.2\left(\mathrm{~d},{ }^{3} J_{\mathrm{C}-\mathrm{P}}=6.9 \mathrm{~Hz}\right), 37.5,63.1\left(\mathrm{~d},{ }^{2} J_{\mathrm{C}-\mathrm{P}}=5.9\right.$ $\mathrm{Hz}), 93.5\left(\mathrm{~d},{ }^{3} J_{\mathrm{C}-\mathrm{P}}=16.2 \mathrm{~Hz}\right), 103.5\left(\mathrm{~d},{ }^{1} J_{\mathrm{C}-\mathrm{P}}=182.8 \mathrm{~Hz}\right)$, $112.1\left(\mathrm{~d},{ }^{2} J_{\mathrm{C}-\mathrm{F}}=22.9 \mathrm{~Hz}, \operatorname{Ar}-\mathrm{F}\right), 115.4\left(\mathrm{~d},{ }^{2} J_{\mathrm{C}-\mathrm{F}}=23.1 \mathrm{~Hz}\right.$, Ar-F), 116.0, $123.6\left(\mathrm{qd},{ }^{3} J_{\mathrm{C}-\mathrm{P}}=5.2 \mathrm{~Hz},{ }^{1} J_{\mathrm{C}-\mathrm{F}}=275.4 \mathrm{~Hz}\right.$, $\mathrm{CF}_{3}$ ), 124.6, $124.7\left(\mathrm{~d},{ }^{3} J_{\mathrm{C}-\mathrm{F}}=9.5 \mathrm{~Hz}, \mathrm{Ar}-\mathrm{F}\right), 130.1 \sim 130.2$ (m), 133.0, $134.1\left(\mathrm{qd},{ }^{2} J_{\mathrm{C}-\mathrm{P}}=5.0 \mathrm{~Hz},{ }^{2} J_{\mathrm{C}-\mathrm{F}}=32.3 \mathrm{~Hz}\right)$, $148.2,148.3,155.8\left(\mathrm{~d},{ }^{2} J_{\mathrm{C}-\mathrm{P}}=10.1 \mathrm{~Hz}\right), 164.4\left(\mathrm{~d},{ }^{1} J_{\mathrm{C}-\mathrm{F}}=\right.$ $250.4 \mathrm{~Hz}, \mathrm{Ar}-\mathrm{F}) ;{ }^{19} \mathrm{~F}$ NMR $\left(564 \mathrm{MHz}, \mathrm{CDCl}_{3}\right) \delta:-108.5$ (s, ArF), $-55.0\left(\mathrm{~s}, \mathrm{CF}_{3}\right)$; IR (KBr) v: 3302, 3228, 2906, 2208, 1611, 1405, 1284, 1175, 1047, 1022, $764 \mathrm{~cm}^{-1}$; MS (ESI) $m / z: 429(\mathrm{M}+\mathrm{H})^{+}$; HRMS (ESI) calcd for $\mathrm{C}_{19} \mathrm{H}_{18} \mathrm{~F}_{4} \mathrm{~N}_{2} \mathrm{O}_{3} \mathrm{P}(\mathrm{M}+\mathrm{H})^{+}$429.0993, found 429.0988.

3-氨基-7-溴-4-氰基-1-(三氟甲基)- $9 H$-芴-2-炔膦酸 二乙酯(4g): 黄色固体. m.p. 166.2 166.5 ${ }^{\circ} \mathrm{C} ;{ }^{1} \mathrm{H}$ NMR $\left(500 \mathrm{MHz}, \mathrm{CDCl}_{3}\right) \delta: 1.36(\mathrm{t}, J=7.0 \mathrm{~Hz}, 6 \mathrm{H}), 4.03(\mathrm{~s}, 2 \mathrm{H})$, $4.10 \sim 4.27(\mathrm{~m}, 4 \mathrm{H}), 7.16$ (brs, $2 \mathrm{H}), 7.59 \sim 7.61(\mathrm{~m}, 1 \mathrm{H})$, $7.72(\mathrm{~s}, 1 \mathrm{H}), 8.31 \sim 8.32(\mathrm{~m}, 1 \mathrm{H}) ;{ }^{13} \mathrm{C} \mathrm{NMR}(125 \mathrm{MHz}$, $\left.\mathrm{CDCl}_{3}\right) \delta: 16.2\left(\mathrm{~d},{ }^{3} J_{\mathrm{C}-\mathrm{P}}=7.1 \mathrm{~Hz}\right), 37.3,63.2\left(\mathrm{~d},{ }^{2} J_{\mathrm{C}-\mathrm{P}}=6.1\right.$ $\mathrm{Hz}), 93.8\left(\mathrm{~d},{ }^{3} J_{\mathrm{C}-\mathrm{P}}=16.3 \mathrm{~Hz}\right), 104.4\left(\mathrm{~d},{ }^{1} J_{\mathrm{C}-\mathrm{P}}=182.4 \mathrm{~Hz}\right)$, $115.9,123.6\left(\mathrm{qd},{ }^{3} J_{\mathrm{C}-\mathrm{P}}=5.1 \mathrm{~Hz},{ }^{1} J_{\mathrm{C}-\mathrm{F}}=275.3 \mathrm{~Hz}, \mathrm{CF}_{3}\right)$, $124.2,125.2,128.1,129.9 \sim 130.0(\mathrm{~m}), 131.0,134.4(\mathrm{qd}$, $\left.{ }^{2} J_{\mathrm{C}-\mathrm{P}}=5.0 \mathrm{~Hz},{ }^{2} J_{\mathrm{C}-\mathrm{F}}=32.3 \mathrm{~Hz}\right), 135.8,147.3,147.4,148.2$, $155.8\left(\mathrm{~d},{ }^{2} J_{\mathrm{C}-\mathrm{P}}=10.4 \mathrm{~Hz}\right) ;{ }^{19} \mathrm{~F}$ NMR $\left(376 \mathrm{MHz}, \mathrm{CDCl}_{3}\right) \delta$ : -54.9 (s, $\left.\mathrm{CF}_{3}\right)$; IR (KBr) v: 3293, 3197, 2914, 2848, 2211, 1612, 1401, 1283, 1188, 1044, 1021, $771 \mathrm{~cm}^{-1}$; MS (ESI) $m / z: 489(\mathrm{M}+\mathrm{H})^{+}$; HRMS (ESI) calcd for $\mathrm{C}_{19} \mathrm{H}_{18} \mathrm{Br}-$ $\mathrm{F}_{3} \mathrm{~N}_{2} \mathrm{O}_{3} \mathrm{P}(\mathrm{M}+\mathrm{H})^{+}$489.0192, found 489.0190.

3-氨基-4-氰基-8-甲基-1-(三氟甲基)- $9 H$-芴-2-炔膦 酸二乙酯(4h): 黄色固体. m.p. $189.9 \sim 190.4{ }^{\circ} \mathrm{C} ;{ }^{1} \mathrm{H}$ NMR (500 MHz, $\left.\mathrm{CDCl}_{3}\right) \delta: 1.36(\mathrm{t}, J=7.3 \mathrm{~Hz}, 6 \mathrm{H}), 2.43$ (s, 3H), $3.91(\mathrm{~s}, 2 \mathrm{H}), 4.11 \sim 4.27(\mathrm{~m}, 4 \mathrm{H}), 7.13$ (brs, 2H), $7.30 \sim 7.31(\mathrm{~m}, 1 \mathrm{H}), 7.38 \sim 7.41(\mathrm{~m}, 1 \mathrm{H}), 8.30 \sim 8.32(\mathrm{~m}$, $1 \mathrm{H}) ;{ }^{13} \mathrm{C}$ NMR $\left(125 \mathrm{MHz}, \mathrm{CDCl}_{3}\right) \delta: 16.2\left(\mathrm{~d},{ }^{3} J_{\mathrm{C}-\mathrm{P}}=7.0\right.$ $\mathrm{Hz}), 18.8,36.4,63.0\left(\mathrm{~d},{ }^{2} J_{\mathrm{C}-\mathrm{P}}=5.9 \mathrm{~Hz}\right), 93.9\left(\mathrm{~d},{ }^{3} J_{\mathrm{C}-\mathrm{P}}=\right.$ $16.2 \mathrm{~Hz}), 103.5\left(\mathrm{~d},{ }^{1} J_{\mathrm{C}-\mathrm{P}}=183.0 \mathrm{~Hz}\right), 116.1,120.5,123.7$ $\left(\mathrm{qd},{ }^{3} J_{\mathrm{C}-\mathrm{P}}=5.3 \mathrm{~Hz},{ }^{1} J_{\mathrm{C}-\mathrm{F}}=275.3 \mathrm{~Hz}, \mathrm{CF}_{3}\right), 128.0,130.4 \sim$ $130.5(\mathrm{~m}), 131.5,134.1\left(\mathrm{qd},{ }^{2} J_{\mathrm{C}-\mathrm{P}}=5.0 \mathrm{~Hz},{ }^{2} J_{\mathrm{C}-\mathrm{F}}=32.3\right.$ $\mathrm{Hz}), 136.4,144.3,149.7,155.7\left(\mathrm{~d},{ }^{2} J_{\mathrm{C}-\mathrm{P}}=10.5 \mathrm{~Hz}\right) ;{ }^{19} \mathrm{~F}$ NMR $\left(376 \mathrm{MHz}, \mathrm{CDCl}_{3}\right) \delta:-55.5\left(\mathrm{~s}, \mathrm{CF}_{3}\right)$; IR (KBr) v: 3301, 3199, 2911, 2851, 2210, 1629, 1397, 1286, 1163, 1121, 1020, $762 \mathrm{~cm}^{-1}$; MS (ESI) m/z: $425(\mathrm{M}+\mathrm{H})^{+}$; HRMS (ESI) calcd for $\mathrm{C}_{20} \mathrm{H}_{21} \mathrm{~F}_{3} \mathrm{~N}_{2} \mathrm{O}_{3} \mathrm{P} \quad(\mathrm{M}+\mathrm{H})$ 425.1236, found 425.1238.

3-氨基-4-氰基-7-甲基-1-(三氟甲基)-9H-芴-2-炔膦 酸二乙酯(4i): 黄色固体. m.p. $172.3 \sim 172.8{ }^{\circ} \mathrm{C} ;{ }^{1} \mathrm{H}$ NMR (500 MHz, $\left.\mathrm{CDCl}_{3}\right) \delta: 1.36$ (t, $\left.J=7.0 \mathrm{~Hz}, 6 \mathrm{H}\right), 2.46$ (s, 3H), $3.98(\mathrm{~s}, 2 \mathrm{H}), 4.10 \sim 4.27(\mathrm{~m}, 4 \mathrm{H}), 7.11$ (brs, 2H), $7.26 \sim 7.27(\mathrm{~m}, 1 \mathrm{H}), 7.36(\mathrm{~s}, 1 \mathrm{H}), 8.31 \sim 8.32(\mathrm{~m}, 1 \mathrm{H}) ;{ }^{13} \mathrm{C}$ NMR (125 MHz, $\left.\mathrm{CDCl}_{3}\right) \delta: 16.2\left(\mathrm{~d},{ }^{3} J_{\mathrm{C}-\mathrm{P}}=6.5 \mathrm{~Hz}\right), 22.0$, $37.3,63.0\left(\mathrm{~d},{ }^{2} J_{\mathrm{C}-\mathrm{P}}=5.6 \mathrm{~Hz}\right), 93.4\left(\mathrm{~d},{ }^{3} J_{\mathrm{C}-\mathrm{P}}=16.4 \mathrm{~Hz}\right)$, 
$102.8\left(\mathrm{~d},{ }^{1} J_{\mathrm{C}-\mathrm{P}}=183.0 \mathrm{~Hz}\right), 116.2,122.7,123.7\left(\mathrm{qd},{ }^{3} J_{\mathrm{C}-\mathrm{P}}=\right.$ $\left.5.2 \mathrm{~Hz},{ }^{1} J_{\mathrm{C}-\mathrm{F}}=275.3 \mathrm{~Hz}, \mathrm{CF}_{3}\right), 125.3,128.7,130.4 \sim 130.5$ (m), 134.2, $133.9\left(\mathrm{qd},{ }^{2} J_{\mathrm{C}-\mathrm{P}}=5.0 \mathrm{~Hz},{ }^{2} J_{\mathrm{C}-\mathrm{F}}=32.4 \mathrm{~Hz}\right)$, 141.3, 145.9, 149.5, 155.7 (d, $\left.{ }^{2} J_{\mathrm{C}-\mathrm{P}}=10.4 \mathrm{~Hz}\right) ;{ }^{19} \mathrm{~F}$ NMR $\left(470 \mathrm{MHz}, \mathrm{CDCl}_{3}\right) \delta:-54.9\left(\mathrm{~s}, \mathrm{CF}_{3}\right)$; IR (KBr) v: 3391, 3217, 2911, 2212, 1638, 1400, 1286, 1165, 1147, 1043, 1016, $773 \mathrm{~cm}^{-1}$; MS (ESI) $m / z: 425(\mathrm{M}+\mathrm{H})^{+}$; HRMS (DART) calcd for $\mathrm{C}_{20} \mathrm{H}_{21} \mathrm{~F}_{3} \mathrm{~N}_{2} \mathrm{O}_{3} \mathrm{P}(\mathrm{M}+\mathrm{H})^{+}$425.1236, found 425.1237 .

3-氨基-4-氯基-6-甲基-1-(三氟甲基)-9H-芴-2-炔膦 酸二乙酯 $(\mathbf{4 j})$ : 黄色固体. m.p. $126.2 \sim 126.8{ }^{\circ} \mathrm{C} ;{ }^{1} \mathrm{H}$ NMR (500 MHz, $\left.\mathrm{CDCl}_{3}\right) \delta: 1.38(\mathrm{t}, J=7.3 \mathrm{~Hz}, 6 \mathrm{H}), 2.51$ (s, 3H), $4.03(\mathrm{~s}, 2 \mathrm{H}), 4.13 \sim 4.30(\mathrm{~m}, 4 \mathrm{H}), 7.16$ (brs, 2H), $7.33 \sim 7.34(\mathrm{~m}, 1 \mathrm{H}), 7.47 \sim 7.48(\mathrm{~m}, 1 \mathrm{H}), 8.30(\mathrm{~s}, 1 \mathrm{H}) ;{ }^{13} \mathrm{C}$ NMR (125 MHz, $\left.\mathrm{CDCl}_{3}\right) \delta: 16.2\left(\mathrm{~d},{ }^{3} J_{\mathrm{C}-\mathrm{P}}=6.6 \mathrm{~Hz}\right), 21.7$, $37.1,63.0\left(\mathrm{~d},{ }^{2} J_{\mathrm{C}-\mathrm{P}}=5.6 \mathrm{~Hz}\right), 93.7\left(\mathrm{~d},{ }^{3} J_{\mathrm{C}-\mathrm{P}}=16.3 \mathrm{~Hz}\right)$, $103.4\left(\mathrm{~d},{ }^{1} J_{\mathrm{C}-\mathrm{P}}=183.0 \mathrm{~Hz}\right), 116.2,123.3,123.7\left(\mathrm{qd},{ }^{3} J_{\mathrm{C}-\mathrm{P}}=\right.$ $\left.5.0 \mathrm{~Hz},{ }^{1} J_{\mathrm{C}-\mathrm{F}}=275.4 \mathrm{~Hz}, \mathrm{CF}_{3}\right), 124.5,130.9 \sim 131.0(\mathrm{~m})$, $131.8,134.1\left(\mathrm{qd},{ }^{2} J_{\mathrm{C}-\mathrm{P}}=5.0 \mathrm{~Hz},{ }^{2} J_{\mathrm{C}-\mathrm{F}}=32.5 \mathrm{~Hz}\right), 136.9$, 137.6, 142.9, 149.4, $155.7\left(\mathrm{~d},{ }^{2} J_{\mathrm{C}-\mathrm{P}}=10.1 \mathrm{~Hz}\right) ;{ }^{19} \mathrm{~F}$ NMR $\left(470 \mathrm{MHz}, \mathrm{CDCl}_{3}\right) \delta:-54.9\left(\mathrm{~s}, \mathrm{CF}_{3}\right)$; IR (KBr) v: 3306, 3194, 2911, 2217, 1628, 1396, 1282, 1159, 1124, 1046, 1019, $765 \mathrm{~cm}^{-1}$; MS (ESI) m/z: $425[(\mathrm{M}+\mathrm{H})]^{+}$; HRMS (ESI) calcd for $\mathrm{C}_{20} \mathrm{H}_{21} \mathrm{~F}_{3} \mathrm{~N}_{2} \mathrm{O}_{3} \mathrm{P}(\mathrm{M}+\mathrm{H})^{+} 425.1236$, found 425.1241 .

3-氨基-4-氰基-6-甲氧基-1-(三氟甲基)-9H-芴-2-炔 膦酸二乙酯(4k): 黄色固体. m.p. $160.8 \sim 161.3{ }^{\circ} \mathrm{C} ;{ }^{1} \mathrm{H}$ NMR (400 MHz, $\left.\mathrm{CDCl}_{3}\right) \delta: 1.35(\mathrm{t}, J=7.0 \mathrm{~Hz}, 6 \mathrm{H}), 3.90$ $(\mathrm{s}, 3 \mathrm{H}), 3.96(\mathrm{~s}, 2 \mathrm{H}), 4.08 \sim 4.27(\mathrm{~m}, 4 \mathrm{H}), 7.05 \sim 7.07(\mathrm{~m}$, 1H), 7.12 (brs, 2H), $7.43 \sim 7.45(\mathrm{~m}, 1 \mathrm{H}), 7.98 \sim 7.99(\mathrm{~m}$, $1 \mathrm{H}) ;{ }^{13} \mathrm{C}$ NMR $\left(125 \mathrm{MHz}, \mathrm{CDCl}_{3}\right) \delta: 16.2\left(\mathrm{~d},{ }^{3} J_{\mathrm{C}-\mathrm{P}}=7.0\right.$ $\mathrm{Hz}), 36.8,55.7,63.0\left(\mathrm{~d},{ }^{2} J_{\mathrm{C}-\mathrm{P}}=5.8 \mathrm{~Hz}\right), 93.7\left(\mathrm{~d},{ }^{3} J_{\mathrm{C}-\mathrm{P}}=\right.$ $16.3 \mathrm{~Hz}), 103.6\left(\mathrm{~d},{ }^{1} J_{\mathrm{C}-\mathrm{P}}=183.0 \mathrm{~Hz}\right), 106.4,116.1,118.6$, $123.7\left(\mathrm{dq},{ }^{3} J_{\mathrm{C}-\mathrm{P}}=5.0 \mathrm{~Hz},{ }^{1} J_{\mathrm{C}-\mathrm{F}}=275.4 \mathrm{~Hz}, \mathrm{CF}_{3}\right), 125.4$, $131.5 \sim 131.6(\mathrm{~m}), 134.0\left(\mathrm{qd},{ }^{2} J_{\mathrm{C}-\mathrm{P}}=5.0 \mathrm{~Hz},{ }^{2} J_{\mathrm{C}-\mathrm{F}}=32.2\right.$ $\mathrm{Hz}), 137.9,138.0,149.3,155.7$ (d, $\left.{ }^{2} J_{\mathrm{C}-\mathrm{P}}=10.3 \mathrm{~Hz}\right), 160.0$; ${ }^{19} \mathrm{~F}$ NMR $\left(376 \mathrm{MHz}, \mathrm{CDCl}_{3}\right) \delta:-54.8\left(\mathrm{~s}, \mathrm{CF}_{3}\right)$; IR (KBr) $v: \quad 3292,3215,2995,2914,2218,1610,1396,1287$, 1169, 1046, 1015, $794 \mathrm{~cm}^{-1}$; MS (ESI) $m / z: 441(\mathrm{M}+\mathrm{H})^{+}$; HRMS (ESI) calcd for $\mathrm{C}_{20} \mathrm{H}_{21} \mathrm{~F}_{3} \mathrm{~N}_{2} \mathrm{O}_{4} \mathrm{P}[(\mathrm{M}+\mathrm{H})]^{+}$ 441.1195, found 441.1188.

3-氨基-4-氰基-1-(五氟乙基)- $9 H$-芴-2-炔膦酸二乙 酯(4l): 黄色固体. m.p. 168.6 169.1 ${ }^{\circ} \mathrm{C} ;{ }^{1} \mathrm{H}$ NMR (400 $\left.\mathrm{MHz}, \mathrm{CDCl}_{3}\right) \delta: 1.34(\mathrm{t}, J=7.0 \mathrm{~Hz}, 6 \mathrm{H}), 4.07(\mathrm{~s}, 2 \mathrm{H})$, $4.10 \sim 4.28(\mathrm{~m}, 4 \mathrm{H}), 7.30$ (brs, $2 \mathrm{H}), 7.45 \sim 7.51(\mathrm{~m}, 2 \mathrm{H})$,
$7.55 \sim 7.57(\mathrm{~m}, 1 \mathrm{H}), 8.48 \sim 8.50(\mathrm{~m}, 1 \mathrm{H}) ;{ }^{13} \mathrm{C}$ NMR $(125$ $\left.\mathrm{MHz}, \mathrm{CDCl}_{3}\right) \delta: 16.1\left(\mathrm{~d},{ }^{3} J_{\mathrm{C}-\mathrm{P}}=6.4 \mathrm{~Hz}\right), 37.6,62.9(\mathrm{~d}$, $\left.{ }^{2} J_{\mathrm{C}-\mathrm{P}}=6.1 \mathrm{~Hz}\right), 94.2\left(\mathrm{~d},{ }^{3} J_{\mathrm{C}-\mathrm{P}}=16.8 \mathrm{~Hz}\right), 104.8\left(\mathrm{~d},{ }^{1} J_{\mathrm{C}-\mathrm{P}}=\right.$ $185.4 \mathrm{~Hz}), 112.2 \sim 117.3\left(\mathrm{~m}, \mathrm{CF}_{2}\right), 116.0,119.6\left(\mathrm{qt},{ }^{1} J_{\mathrm{C}-\mathrm{F}}=\right.$ $\left.287.4 \mathrm{~Hz},{ }^{2} J_{\mathrm{C}-\mathrm{F}}=37.4 \mathrm{~Hz}, \mathrm{CF}_{3}\right), 122.9,124.5,127.5,130.5$, $131.4 \sim 131.5(\mathrm{~m}), 132.8 \sim 133.2(\mathrm{~m}), 136.3,145.5 \sim 145.6$ (m), 149.1, $155.6\left(\mathrm{~d},{ }^{2} J_{\mathrm{C}-\mathrm{P}}=10.9 \mathrm{~Hz}\right) ;{ }^{19} \mathrm{~F}$ NMR $(376 \mathrm{MHz}$, $\left.\mathrm{CDCl}_{3}\right) \delta$ : $-78.1\left(\mathrm{~s}, \mathrm{CF}_{3}\right),-101.3\left(\mathrm{~s}, \mathrm{CF}_{2}\right)$; IR $(\mathrm{KBr}) v$ : 3389, 3215, 2924, 2851, 2214, 1638, 1395, 1271, 1178, 1039, 1016, $761 \mathrm{~cm}^{-1}$; MS (ESI) $\mathrm{m} / z: 461(\mathrm{M}+\mathrm{H})^{+}$; HRMS (ESI) calcd. for $\mathrm{C}_{20} \mathrm{H}_{19} \mathrm{~F}_{5} \mathrm{~N}_{2} \mathrm{O}_{3} \mathrm{P} \quad(\mathrm{M}+\mathrm{H}){ }^{+}$ 461.1060, found 461.1052 .

3-氨基-4-氰基-7-甲基-1-(五氟乙基)- $9 H$-芴-2-炔膦 酸二乙酯 $(4 \mathrm{~m})$ : 黄色固体. m.p. $178.1 \sim 178.8{ }^{\circ} \mathrm{C} ;{ }^{1} \mathrm{H}$ NMR (400 MHz, $\left.\mathrm{CDCl}_{3}\right) \delta: 1.34(\mathrm{t}, J=7.2 \mathrm{~Hz}, 6 \mathrm{H}), 2.45$ $(\mathrm{s}, 3 \mathrm{H}), 4.01(\mathrm{~s}, 2 \mathrm{H}), 4.08 \sim 4.28(\mathrm{~m}, 4 \mathrm{H}), 7.26(\mathrm{~s}, 1 \mathrm{H})$, 7.28 (brs, 2H), 7.36 (s, 1H), 8.33 8.35 (m, 1H); ${ }^{13} \mathrm{C} \mathrm{NMR}$ $\left(125 \mathrm{MHz}, \mathrm{CDCl}_{3}\right) \delta: 16.2\left(\mathrm{~d},{ }^{3} J_{\mathrm{C}-\mathrm{P}}=6.4 \mathrm{~Hz}\right), 22.0,37.5$, $63.0\left(\mathrm{~d},{ }^{2} J_{\mathrm{C}-\mathrm{P}}=6.0 \mathrm{~Hz}\right), 93.9\left(\mathrm{~d},{ }^{3} J_{\mathrm{C}-\mathrm{P}}=16.8 \mathrm{~Hz}\right), 104.1(\mathrm{~d}$, $\left.{ }^{1} J_{\mathrm{C}-\mathrm{P}}=185.9 \mathrm{~Hz}\right), 112.6 \sim 117.1\left(\mathrm{~m}, \mathrm{CF}_{2}\right), 116.3,119.8$ (qt, $\left.{ }^{1} J_{\mathrm{C}-\mathrm{F}}=287.4 \mathrm{~Hz},{ }^{2} J_{\mathrm{C}-\mathrm{F}}=37.5 \mathrm{~Hz}, \mathrm{CF}_{3}\right), 122.9,125.2,128.6$, $131.5 \sim 131.7(\mathrm{~m}), 132.7 \sim 133.1(\mathrm{~m}), 133.9,141.4,146.1$, 149.5, $155.7\left(\mathrm{~d},{ }^{2} J_{\mathrm{C}-\mathrm{P}}=10.9 \mathrm{~Hz}\right) ;{ }^{19} \mathrm{~F}$ NMR $(376 \mathrm{MHz}$, $\left.\mathrm{CDCl}_{3}\right) \delta:-78.2\left(\mathrm{~s}, \mathrm{CF}_{3}\right),-101.3\left(\mathrm{~s}, \mathrm{CF}_{2}\right)$; IR (KBr) v: 3280, 3212, 2909, 2213, 1628, 1394, 1275, 1205, 1047, 1016, $768 \mathrm{~cm}^{-1}$; MS (ESI) m/z: $475(\mathrm{M}+\mathrm{H})^{+}$; HRMS (ESI) calcd. for $\mathrm{C}_{21} \mathrm{H}_{21} \mathrm{~F}_{5} \mathrm{~N}_{2} \mathrm{O}_{3} \mathrm{P}(\mathrm{M}+\mathrm{H})^{+} 475.1212$, found 475.1206 .

3-氨基-4-氰基-6-甲氧基-1-(五氟乙基)-9H-芴-2-炔 膦酸二乙酯(4n): 黄色固体. m.p. $133.8 \sim 134.1{ }^{\circ} \mathrm{C} ;{ }^{1} \mathrm{H}$ NMR (500 MHz, $\left.\mathrm{CDCl}_{3}\right) \delta: 1.35(\mathrm{t}, J=7.0 \mathrm{~Hz}, 6 \mathrm{H}), 3.92$ $(\mathrm{s}, 3 \mathrm{H}), 4.00(\mathrm{~s}, 2 \mathrm{H}), 4.10 \sim 4.28(\mathrm{~m}, 4 \mathrm{H}), 7.07 \sim 7.09(\mathrm{~m}$, 1H), 7.30 (brs, 2H), $7.43 \sim 7.45(\mathrm{~m}, 1 \mathrm{H}), 8.02(\mathrm{~s}, 1 \mathrm{H}) ;{ }^{13} \mathrm{C}$ NMR (125 MHz, $\left.\mathrm{CDCl}_{3}\right) \delta: 16.2\left(\mathrm{~d},{ }^{3} J_{\mathrm{C}-\mathrm{P}}=6.5 \mathrm{~Hz}\right), 37.0$, $55.7,63.0\left(\mathrm{~d},{ }^{2} J_{\mathrm{C}-\mathrm{P}}=6.1 \mathrm{~Hz}\right), 94.1\left(\mathrm{~d},{ }^{3} J_{\mathrm{C}-\mathrm{P}}=16.8 \mathrm{~Hz}\right)$, $104.8\left(\mathrm{~d},{ }^{1} J_{\mathrm{C}-\mathrm{P}}=185.4 \mathrm{~Hz}\right), 106.5,112.3 \sim 117.4\left(\mathrm{~m}, \mathrm{CF}_{2}\right)$, $116.3,118.8,119.7\left(\mathrm{qt},{ }^{1} J_{\mathrm{C}-\mathrm{F}}=287.5 \mathrm{~Hz},{ }^{2} J_{\mathrm{C}-\mathrm{F}}=37.3 \mathrm{~Hz}\right.$, $\left.\mathrm{CF}_{3}\right), 125.3,132.6 \sim 132.7(\mathrm{~m}), 132.9 \sim 133.3(\mathrm{~m}), 137.6$, $138.1 \sim 138.2(\mathrm{~m}), 149.3,155.7\left(\mathrm{~d},{ }^{2} J_{\mathrm{C}-\mathrm{P}}=10.9 \mathrm{~Hz}\right), 159.6$; ${ }^{19} \mathrm{~F}$ NMR $\left(376 \mathrm{MHz}, \mathrm{CDCl}_{3}\right) \delta$ : $-78.0\left(\mathrm{~s}, \mathrm{CF}_{3}\right),-101.2$ (s, $\mathrm{CF}_{2}$ ); IR (KBr) v: 3279, 3213, 2924, 2215, 1610, 1388, 1281, 1204, 1043, 1015, $794 \mathrm{~cm}^{-1}$; MS (ESI) $\mathrm{m} / z$ : 491 $(\mathrm{M}+\mathrm{H})^{+}$; HRMS (ESI) calcd for $\mathrm{C}_{21} \mathrm{H}_{21} \mathrm{~F}_{5} \mathrm{~N}_{2} \mathrm{O}_{4} \mathrm{P}(\mathrm{M}+\mathrm{H})^{+}$ 491.1162, found 491.1156 .

3-氨基-4-氰基-1-(七氟正丙基)- $9 H$-芴-2-炔膦酸二 
乙酯(4o): 黄色固体; m.p. 172.1 172.5 ${ }^{\circ} \mathrm{C} ;{ }^{1} \mathrm{H}$ NMR $\left(500 \mathrm{MHz}, \mathrm{CDCl}_{3}\right) \delta: 1.34(\mathrm{t}, J=7.3 \mathrm{~Hz}, 6 \mathrm{H}), 4.09(\mathrm{~s}, 2 \mathrm{H})$, $4.10 \sim 4.28(\mathrm{~m}, 4 \mathrm{H}), 7.37$ (brs, $2 \mathrm{H}), 7.47 \sim 7.52(\mathrm{~m}, 2 \mathrm{H})$, $7.56 \sim 7.57(\mathrm{~m}, 1 \mathrm{H}), 8.51 \sim 8.52(\mathrm{~m}, 1 \mathrm{H}) ;{ }^{13} \mathrm{C}$ NMR $(125$ $\left.\mathrm{MHz}, \mathrm{CDCl}_{3}\right) \delta: 16.3\left(\mathrm{~d},{ }^{3} J_{\mathrm{C}-\mathrm{P}}=5.9 \mathrm{~Hz}\right), 38.2,63.0$ (d, $\left.{ }^{2} J_{\mathrm{C}-\mathrm{P}}=6.0 \mathrm{~Hz}\right), 94.5\left(\mathrm{~d},{ }^{3} J_{\mathrm{C}-\mathrm{P}}=16.9 \mathrm{~Hz}\right), 105.3\left(\mathrm{~d},{ }^{1} J_{\mathrm{C}-\mathrm{P}}=\right.$ $185.3 \mathrm{~Hz}), 107.4 \sim 112.5\left(\mathrm{~m}, \mathrm{CF}_{2}\right), 114.4 \sim 116.8\left(\mathrm{~m}, \mathrm{CF}_{2}\right)$, 116.3, 116.8 121.6 (m, $\left.\mathrm{CF}_{3}\right), 123.1,124.6,127.6,130.7$, $132.2 \sim 132.4(\mathrm{~m}), 132.5 \sim 132.6(\mathrm{~m}), 136.4,145.8 \sim 145.9$ (m), 149.3, $155.8\left(\mathrm{~d},{ }^{2} J_{\mathrm{C}-\mathrm{P}}=10.9 \mathrm{~Hz}\right) ;{ }^{19} \mathrm{~F}$ NMR $(470 \mathrm{MHz}$, $\left.\mathrm{CDCl}_{3}\right) \delta:-80.0\left(\mathrm{t}, J=10.6 \mathrm{~Hz}, \mathrm{CF}_{3}\right),-100.7\left(\mathrm{~s}, \mathrm{CF}_{2}\right)$, $-120.7\left(\mathrm{~s}, \mathrm{CF}_{2}\right)$; IR (KBr) v: 3295, 3199, 2911, 2213, 1612, 1391, 1268, 1222, 1196, 1046, 1009, $743 \mathrm{~cm}^{-1}$; MS (ESI) $m / z: 511(\mathrm{M}+\mathrm{H})^{+}$; HRMS (ESI) calcd for $\mathrm{C}_{21} \mathrm{H}_{19} \mathrm{~F}_{7} \mathrm{~N}_{2} \mathrm{O}_{3} \mathrm{P}(\mathrm{M}+\mathrm{H})^{+}$511.1025, found 511.1020.

3-氨基-4-氰基-7-甲基-1-(七氟正丙基)- $9 H$-芴-2-炔 膦酸二乙酯 $(4 p)$ : 黄色固体. m.p. $178.2 \sim 178.6{ }^{\circ} \mathrm{C} ;{ }^{1} \mathrm{H}$ NMR (500 MHz, $\left.\mathrm{CDCl}_{3}\right) \delta: 1.34(\mathrm{t}, J=7.0 \mathrm{~Hz}, 6 \mathrm{H}), 2.47$ $(\mathrm{s}, 3 \mathrm{H}), 4.06(\mathrm{~s}, 2 \mathrm{H}), 4.08 \sim 4.27(\mathrm{~m}, 4 \mathrm{H}), 7.27(\mathrm{~s}, 1 \mathrm{H})$, 7.28 (brs, $2 \mathrm{H}), 7.36$ (s, 1H), 8.35 8.37 (m, 1H); ${ }^{13} \mathrm{C} \mathrm{NMR}$ $\left(125 \mathrm{MHz}, \mathrm{CDCl}_{3}\right) \delta: 16.1\left(\mathrm{~d},{ }^{3} J_{\mathrm{C}-\mathrm{P}}=6.1 \mathrm{~Hz}\right), 21.8,37.8$, $62.8\left(\mathrm{~d},{ }^{2} J_{\mathrm{C}-\mathrm{P}}=6.3 \mathrm{~Hz}\right), 94.0\left(\mathrm{~d},{ }^{3} J_{\mathrm{C}-\mathrm{P}}=16.8 \mathrm{~Hz}\right), 104.3(\mathrm{~d}$, $\left.{ }^{1} J_{\mathrm{C}-\mathrm{P}}=185.6 \mathrm{~Hz}\right), 107.3 \sim 112.4\left(\mathrm{~m}, \mathrm{CF}_{2}\right), 114.3 \sim 116.7$ $\left(\mathrm{m}, \mathrm{CF}_{2}\right), 116.2,116.9 \sim 121.8\left(\mathrm{~m}, \mathrm{CF}_{3}\right), 122.7,125.0$, $128.5,132.0 \sim 132.1(\mathrm{~m}), 132.2,133.7,141.3,146.1$, 149.4, $155.6\left(\mathrm{~d},{ }^{2} J_{\mathrm{C}-\mathrm{P}}=10.9 \mathrm{~Hz}\right) ;{ }^{19} \mathrm{~F}$ NMR $(470 \mathrm{MHz}$, $\left.\mathrm{CDCl}_{3}\right) \delta:-80.0\left(\mathrm{t}, J=10.3 \mathrm{~Hz}, \mathrm{CF}_{3}\right),-100.7\left(\mathrm{~s}, \mathrm{CF}_{2}\right)$, $-120.8\left(\mathrm{~s}, \mathrm{CF}_{2}\right)$; IR (KBr) v: 3270, 3191, 2903, 2210, 1618, 1393, 1220, 1205, 1046, 1021, $760 \mathrm{~cm}^{-1}$; MS (ESI) $m / z: 525(\mathrm{M}+\mathrm{H})^{+}$; HRMS (ESI) calcd for $\mathrm{C}_{22} \mathrm{H}_{21} \mathrm{~F}_{7} \mathrm{~N}_{2} \mathrm{O}_{3} \mathrm{P}$ $(\mathrm{M}+\mathrm{H})^{+}$525.1184, found 525.1178.

3-氨基-4-氰基-6-甲氧基-1-(七氟正丙基)- $9 H$-芴-2炔膦酸二乙酯 $(4 q)$ : 黄色固体; m.p. 154.9 155.2 ${ }^{\circ} \mathrm{C} ;{ }^{1} \mathrm{H}$ NMR (400 MHz, $\left.\mathrm{CDCl}_{3}\right) \delta: 1.33(\mathrm{t}, J=7.0 \mathrm{~Hz}, 6 \mathrm{H}), 3.91$ $(\mathrm{s}, 3 \mathrm{H}), 4.02(\mathrm{~s}, 2 \mathrm{H}), 4.06 \sim 4.27(\mathrm{~m}, 4 \mathrm{H}), 7.05 \sim 7.08(\mathrm{~m}$, $1 \mathrm{H}), 7.35$ (brs, $2 \mathrm{H}), 7.42 \sim 7.44(\mathrm{~m}, 1 \mathrm{H}), 8.02(\mathrm{~s}, 1 \mathrm{H}) ;{ }^{13} \mathrm{C}$ NMR (125 MHz, $\left.\mathrm{CDCl}_{3}\right) \delta: 16.3\left(\mathrm{~d},{ }^{3} J_{\mathrm{C}-\mathrm{P}}=5.9 \mathrm{~Hz}\right), 37.4$, $55.7,63.0\left(\mathrm{~d},{ }^{2} J_{\mathrm{C}-\mathrm{P}}=6.0 \mathrm{~Hz}\right), 94.4\left(\mathrm{~d},{ }^{3} J_{\mathrm{C}-\mathrm{P}}=16.8 \mathrm{~Hz}\right)$, $105.1\left(\mathrm{~d},{ }^{1} J_{\mathrm{C}-\mathrm{P}}=185.3 \mathrm{~Hz}\right), 106.4,107.0 \sim 112.5\left(\mathrm{~m}, \mathrm{CF}_{2}\right)$, $115.8\left(\mathrm{~m}, \mathrm{CF}_{2}\right), 116.3,116.8 \sim 121.9\left(\mathrm{~m}, \mathrm{CF}_{3}\right), 118.8$, $125.2,132.2 \sim 132.6(\mathrm{~m}), 133.2 \sim 133.3(\mathrm{~m}), 137.5,138.2$, $149.3,155.7\left(\mathrm{~d},{ }^{2} J_{\mathrm{C}-\mathrm{P}}=10.9 \mathrm{~Hz}\right), 159.5 ;{ }^{19} \mathrm{~F}$ NMR $(376$ $\left.\mathrm{MHz}, \mathrm{CDCl}_{3}\right) \delta:-80.0\left(\mathrm{t}, J=11.5 \mathrm{~Hz}, \mathrm{CF}_{3}\right),-100.6(\mathrm{~s}$, $\left.\mathrm{CF}_{2}\right),-120.6\left(\mathrm{~s}, \mathrm{CF}_{2}\right)$; IR (KBr) v: 3275, 3191, 2923, 2212, 1610, 1386, 1276, 1210, 1195, 1042, 1017, 746 $\mathrm{cm}^{-1}$; MS (ESI) $m / z: 541[(\mathrm{M}+\mathrm{H})]^{+}$; HRMS (ESI) calcd for $\mathrm{C}_{22} \mathrm{H}_{21} \mathrm{~F}_{7} \mathrm{~N}_{2} \mathrm{O}_{4} \mathrm{P}(\mathrm{M}+\mathrm{H})^{+}$541.1132, found 541.1127.

辅助材料(Supporting Information) 全氟烷基取代的 芴基膦酸酯 $\mathbf{4 a} \sim \mathbf{4 q}$ 的核磁共振谱图. 这些材料可以免 费从本刊网站(http://sioc-journal.cn/)上下载.

\section{References}

[1] For selected examples, see: (a) Pearce, A. K.; Rolfe, B. E.; Russell, P. J.; Tse, B. W. C.; Whittaker, A. K.; Fuchs, A. V.; Thurecht, K. J. Polym. Chem. 2014, 5, 6932.

(b) Zeng, W.; Ballard, T. E.; Tkachenko, A. G.; Burns, V. A.; Feldheim, D. L.; Melander, C. Bioorg. Med. Chem. Lett. 2006, 16, 5148. (c) Beutler, U.; Fuenfschilling, P. C.; Steinkemper, A. Org. Process Res. Dev. 2007, 11, 341.

(d) Hicks, L. D.; Hyatt, J. L.; Stoddard, S.; Tsurkan, L.; Edwards, C. C.; Wadkins, R. M.; Potter, P. M. J. Med. Chem. 2009, 52, 3742.

[2] For selected reviews, see: (a) Leclerc, M. J. Polym. Sci., Part A: Polym. Chem. 2001, 39, 2867.

(b) Scherf, U.; List, E. J. W. Adv. Mater. 2002, 14, 477.

(c) Grimsdale, A.; Mallen, K. Adv. Polym. Sci. 2006, 199, 1.

(d) Monkman, A.; Rothe, C.; King, S.; Dias, F. Adv. Polym. Sci. 2008, 212, 187.

(e) Knaapila, M.; Monkman, A. P. Adv. Mater. 2013, 25, 1090.

[3] Liu, B.; Yu, W.; Lai, Y.; Huang, W. Chem. Commun. 2000, 551.

[4] Zhang, F.; Mammo, W.; Andersson, L. Adv. Mater. 2006, 18, 2169.

[5] (a) Fleckenstein, C. A.; Plenio, H. Chem.-Eur. J. 2007, 13, 2701. (b) Bassas, O.; Huuskonen, J.; Rissanen, K.; Koskinen, A. M. P. Eur. J. Org. Chem. 2009, 1340.

(c) Paleo, M. R.; Aurrecoechea, N.; Jung, K.-Y.; Rapoport, H. J. Org. Chem. 2003, 68, 130.

(d) Ding, Y.; Wang, J.; Abboud, K. A.; Xu, Y.; Dolbier, W. R.; Richards, Jr., N. G. J. J. Org. Chem. 2001, 66, 6381.

[6] For recent selected examples, see: (a) Chinnagolla, R. K.; Jeganmohan, M. Org. Lett. 2012, 14, 5246.

(b) Song, J.; Wei, F.; Sun, W.; Li, K.; Tian, Y.; Liu, C.; Li, Y.; Xie, L. Org. Lett. 2015, 17, 2106 .

(c) Sun, F.-L.; Zeng, M.; Gu, Q.; You, S.-L. Chem.-Eur. J. 2009, 15, 8709 .

(d) Peterson, J. J.; Simon, Y. C.; Coughlin, E. B.; Carter, K. R. Chem. Commun. 2009, 4950.

(e) Shabashov, D.; Maldonado, J. R. M.; Daugulis, O. J. Org. Chem. 2008, 73, 7818 .

(f) Barluenga, J.; Trincado, M.; Rubio, E.; Gonz lez, J. M. Angew. Chem., Int. Ed. 2006, 45, 3140.

(g) Wong, K.; Chi, L.; Huang, S.; Liao, Y.; Liu, Y.; Wang, Y. Org. Lett. 2006, 8, 5029.

(h) Wang, S.-G.; Han, L.; Zeng, M.; Sun, F.-L.; Zhang, W.; You, S.-L. Org. Biomol. Chem. 2012, 10, 3202.

[7] For recent selected examples, see: (a) Janina, B.; Thomas, W.; Svenja, T.; Eleni, S.; David, A.; Matthias, R.; Frank, R.; Stephen, A.; Hashmi, K. Adv. Synth. Catal. 2017, 359, 225.

(b) Xu, S.; Chen, R.; Fu, Z.-H.; Zhou, Q.; Zhang, Y.; Wang, J.-B. ACS Catal. 2017, 7, 1993.

(c) Shi, G.-F.; Chen, D.-S.; Jiang, H.; Zhang, Y.; Zhang, Y.-H. Org. Lett. 2016, 18, 2958.

(d) Peng, X.-P.; Luo, H.-W.; Wu, F.-H.; Zhu, D.-H.; Ganesan, A.; Huang, P.; Wen, S.-J. Adv. Synth. Catal. 2017, 359, 1152.

(e) Song, J.; Sun, W.; Li, Y.-L.; Wei, F.-L.; Liu, C.; Qian, Y.; Chen, S.-F. Chem.-Asian J. 2016, 11, 211.

(f) Song, J.; Li, Y.-L.; Sun, W.; Yi, C.-L.; Wu, H.; Wang, H.-T.; Ding, K.-R.; Xiao, K.; Liu, C. New J. Chem. 2016, 40, 9030.

(g) Vergeiner, C.; Ulrich, M.; Li, C.; Liu, X.; Müller, T.; Kräutler, B. Chem.-Eur. J. 2015, 21, 1 . 
(h) Zhou, A.-H.; Pan, F.; Zhu, C.; Ye, L.-W. Chem.-Eur. J. 2015, 21, 10278 .

(i) Song, J.; Sun, W.; Li, Y.; Wei, F.; Liu, C.; Qian, Y.; Chen, S. Chem.-Asian. J. 2016, 11, 211

(j) Zhou, A.-H.; Pan, F.; Zhu, C.; Ye, L.-W. Chem.-Eur. J. 2015, 21, 10278.

[8] For recent selected examples, see: (a) Shi, Z.; Glorius, F. Chem. Sci. 2013, 4, 829 .

(b) Wertz, S.; Leifert, D.; Studer, A. Org. Lett. 2013, 15, 928.

(c) Seo, S.; Slater, M.; Greaney, M. F. Org. Lett. 2012, 14, 2650.

(d) Lockner, J. W.; Dixon, D. D.; Risgaard, R.; Baran, P. S. Org. Lett. 2011, 13, 5628.

(e) Chu, X.-Q.; Xing, Z.-H.; Meng, H.; Xu, X.-P.; Ji, S.-J. Org. Chem. Front. 2016, 3, 165.

[9] (a) Morimoto, K.; Itoh, M.; Hirano, K.; Satoh, T.; Shibata, Y.; Tanaka, K.; Miura, M. Angew. Chem., Int. Ed. 2012, 51, 5359.

(b) Hsiao, C. C.; Lin, Y.-K.; Liu, C.-J.; Wu, T.-C.; Wu, Y.-T. $A d v$. Synth. Catal. 2010, 352, 3267.

(c) Liu, Z.; Tan, H.; Wang, L.; Fu, T.; Xia, Y.; Zhang, Y.; Wang, J. Angew. Chem., Int. Ed. 2015, 54, 3056.

(d) Shi, Y.-B.; Gao, S.-H. Tetrahedron 2016, 72, 1717.

[10] (a) Zhu, J.; Bienayme, H. Multicomponent Reactions, Wiley-VCH, Weinheim, Germany, 2005.

(b) Domling, A. Chem. Rev. 2006, 106, 17.

(c) Tejedor, D.; Garcia-Tellado, F. Chem. Soc. Rev. 2007, 36, 484

(d) Ramon, D. J.; Miguel, Y. Angew. Chem., Int. Ed. 2005, 44, 1602 .

(e) Jiang, B.; Rajale, T.; Wever, W.; Tu, S. J.; Li, G. Chem.-Asian. J. 2010, 5, 2318.

(f) Bello, D.; Ramon, R.; Lavilla, R. Curr. Org. Chem. 2010, 14, 332.

(g) Trost, B. M. Angew. Chem., Int. Ed. Engl. 1995, 34, 259.

(h) Wasilke, J. C.; Obrey, S. J.; Baker, R. T.; Bazan, G. C. Chem. Rev. 2005, 105, 1001.

(i) Tietze, L. F. Chem. Rev. 1996, 96, 115.

(j) Orru, R. V. A.; de Greef, M. Synthesis 2003, 1471. (k) Terrett, N. K. Combinatorial Chemistry, Oxford University Press, New York, 1998

1) Tang, M.; Wu, Y.; Liu, Y.; Cai, M.-Q.; Xia, F.; Liu, S.-Y.; Hu, W.-H. Acta Chim. Sinica 2016, 74, 54 (in Chinese).

(唐敏, 吴永, 刘源, 蔡茂强, 夏飞, 刘顺英, 胡文浩, 化学学报, 2016, 74, 54.)

(m) Wang, J.; Liu, H.-Z.; Wen, R.; Li, J.; Zhu, S.-L. Chin. J. Chem. $\mathbf{2 0 1 7}, 35,1463$.

[11] Sun, Z.-H.; Huang, Q.; Han, J.; He, W.-M.; Chen, J.; Xu, Y.-C.; Deng, H.-M.; Shao, M.; Zhang, H.; Cao, W.-G. Tetrahedron 2018, 74, 2073.

[12] (a) Ojima, I. Fluorine in Medicinal Chemistry and Chemical Biology, John Wiley \& Sons, Chichester, 2009.

(b) Azema, L.; Baron, R.; Ladame, S. Curr. Enzyme Inhib. 2006, 2, 61.

(c) Nagarajan, R.; Pratt, F. R. Biochemistry 2004, 43, 9664.

[13] CCDC 1862491 (4a) contains all crystallographic details of this publication and are available free of charge at www.ccdc.cam.ac. uk/consts/retrieving.html. Or can be ordered from the following address: Cambridge Crystallographic Data Centre, 12 Union Road, GB-Cambridge CB21EZ; fax: + 44 1223336033; or deposit@ccdc.cam.ac.uk. Unit cell parameters (4a): $a=0.7134(16) \mathrm{nm}$, $b=1.146(3) \mathrm{nm}, c=1.266(3) \mathrm{nm} ; \alpha=106.91(3)^{\circ}, \beta=98.99(3)^{\circ}$, $\gamma=91.87(3)^{\circ}$; space group: $P-1$.

[14] (a) Esmaeili, A.; Moradi, A.; Khoddam-Mohammadi, H. Tetrahedron 2010, 66, 3575.

(b) Alizadeh, A.; Hosseini, S.; Sedighian, H.; Bayat, F.; Zhu, Z.; Dusek, M. Tetrahedron 2015, 71, 7885.

(c) Su, W.; Ding, K.; Chen, Z. Tetrahedron Lett. 2009, 50, 636.

[15] Broman, S. L.; Brand, S. L.; Parker, C. R.; Petersen, M. A.; Tortzen, C. G.; Kadziola, A.; Kilsa, K.; Nielsen, M. B. ARKIVOC 2011, ix, 51.

[16] (a) Chambers, R. D.; Holling, D.; Sand-ford, G.; Batsanov, A. S.; Howard, J. A. K. J. Fluorine Chem. 2004, 125, 661.

(b) Nichson, T. E. J. Org. Chem. 1988, 53, 3870. 